REVISIONES

Rev Obstet Ginecol Venez. 2022; 82 (1): 83-97. https://doi.org/10.51288/00820111

\title{
Importancia clínica de la evaluación ecográfica de la translucencia nucal fetal
}

\author{
Eduardo Reyna-Villasmil, ${ }^{1}$ (D) Carlos Briceño-Pérez, ${ }^{2}$ (D) Juan Carlos Briceño-Sanabria. ${ }^{3}$
}

\begin{abstract}
RESUMEN
La translucidez nucal es la región hipoecoica de acumulación de líquido subcutáneo en la parte posterior del cuello fetal, al nivel de la columna cervical, entre la piel y los tejidos blandos. Su medición durante el primer trimestre, forma parte del protocolo de detección prenatal de las trisomías 21, 18 y 13. Entre las 11-13,6 semanas de gestación, su aumento por encima del percentil 95, coloca al feto en mayor riesgo de anomalías cromosómicas y estructurales congénitas. La medición individual de la translucencia nucal es una herramienta de detección parcialmente eficaz pero su combinación con marcadores bioquímicos, aumenta su capacidad de predicción de riesgo de síndrome de Down y otras alteraciones fetales. En los casos de fetos sin aneuploidias, su aumento está asociado con resultantes adversas. El objetivo de este artículo fue revisar la evidencia actual sobre la importancia clínica de la evaluación ecográfica de la translucencia nucal fetal.
\end{abstract}

Palabras clave: Translucencia nucal fetal, Ecografia, Aneuploidias fetales, Cromosomopatías, Cardiopatías congénitas, Alto riesgo prenatal, Diagnóstico prenatal.

\section{Clinical importance of ultrasound evaluation of fetal nuchal translucency} SUMMARY

Nuchal translucency is the hypoechoic region of subcutaneous fluid accumulation at the back of the fetal neck, at the level of the cervical spine, between the skin and soft tissues. Its measurement during the first trimester is part of the prenatal screening protocol for trisomies 21, 18 and 13. Between 11-13.6 weeks of gestation, its increase above the 95th percentile places the fetus at greater risk of congenital chromosomal and structural abnormalities. The individual measurement of nuchal translucency is a partially effective detection tool but its combination with biochemical markers increases its ability to predict the risk of Down syndrome and other fetal alterations. In cases of fetuses without aneuploidies, their increase is associated with adverse results. The objective of this article was to review the current evidence on the clinical significance of ultrasound assessment of fetal nuchal translucency.

Keywords: Fetal nuchal translucency, Ultrasound, Fetal aneuploidies, Chromosomopathies, Congenital heart anomalies, Prenatal high risk, Prenatal diagnosis.

\section{INTRODUCCIÓN}

Durante el primer trimestre del embarazo, una de las evaluaciones ecográficas más útiles para el cribado de aneuploidías fetales es la translucencia nucal (TN)

\footnotetext{
'Doctor en Medicina Clínica. Especialista en Ginecología y Obstetricia. Servicio de Obstetricia y Ginecología. Hospital Central "Dr. Urquinaona". Maracaibo. Estado Zulia. Venezuela. ${ }^{2}$ Doctor en Ciencias Médicas. Especialista en Ginecología y Obstetricia. Profesor Titular del Departamento de Obstetricia y Ginecología, La Universidad del Zulia. Maracaibo, Venezuela. ${ }^{3}$ Especialista en Obstetricia y Ginecología. Médico Adjunto del Servicio de Obstetricia y Ginecología del Hospital San Juan de Dios. Rio Negro, Colombia. Correo para correspondencia: sippenbauch@gmail.com
}

Forma de citar este artículo: Reyna-Villasmil E, Briceño-Pérez C, Briceño-Sanabria JC. Importancia clínica de la evaluación ecográfica de la translucencia nucal fetal. Rev Obstet Ginecol Venez. 2022; 82(1): 83-97. https://doi.org/10.51288/00820111 fetal (1). Esta estructura es el espacio subcutáneo no ecoico, lleno de líquido entre el tejido blando posterior y el tejido cutáneo que cubre la superficie de cuello, y puede observarse al final del primer trimestre del embarazo (Figura 1) (2, 3). Los estudios iniciales indicaban que esta estructura podría predecir el riesgo de aneuploidías fetales cuando su grosor era igual o superior a $3 \mathrm{~mm}$. Desde entonces, es uno de los marcadores ecográficos más útiles e importantes para predecir la presencia de diversas anomalías congénitas y el desarrollo de muerte fetal por cardiopatías congénitas $(\mathrm{CC}) \mathrm{u}$ otras alteraciones genéticas $(4,5)$.

La medición del valor de la TN fetal en forma aislada puede tener una precisión diagnóstico cercana al $50 \%$ 


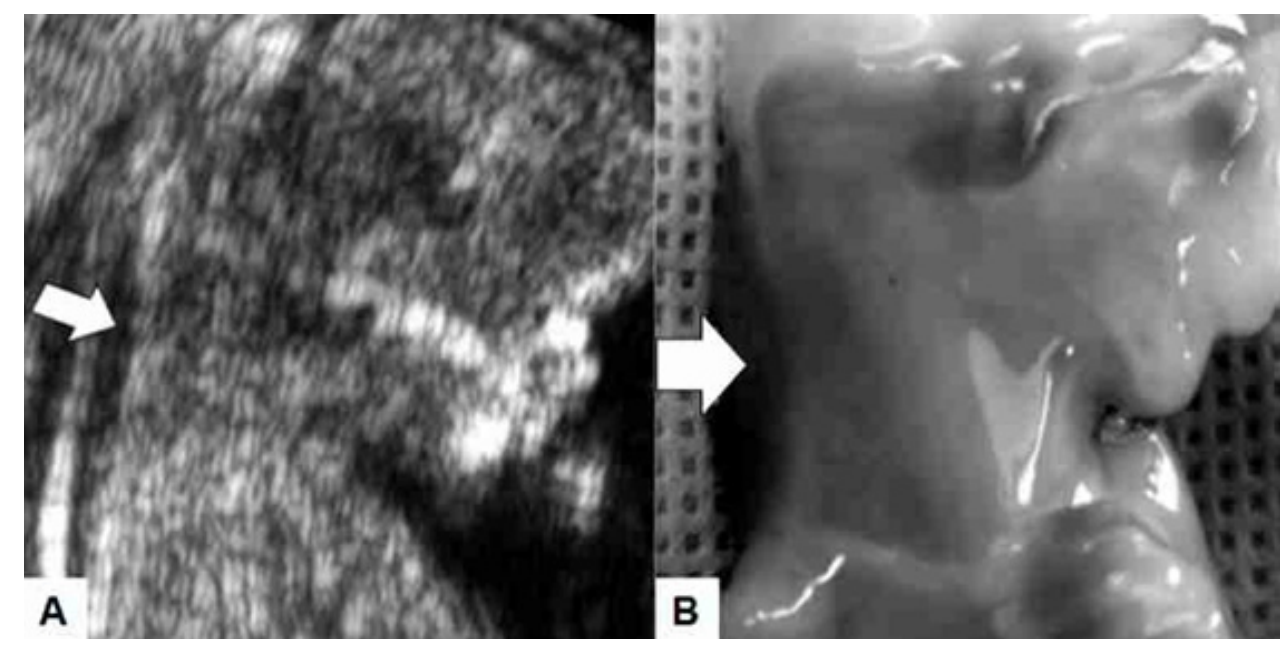

Figura 1. Translucencia nucal fetal. (A) La flecha señala la imagen ecográfica. (B) La flecha señala la imagen anatómica del aumento de la translucencia nucal fetal.

para el síndrome de Down $(\operatorname{SdD})(6,7)$. Durante el primer trimestre del embarazo, al combinarla con marcadores bioquímicos maternos para detección de aneuploidías, esta precisión aumenta hasta aproximadamente $90 \%$ (con una tasa de falsos positivos de $5 \%$ ) (7 - 9). En la evaluación ecográfica prenatal, al permitir el diagnóstico del SdD en una edad gestacional más precoz, comparada con las pruebas bioquímicas, la importancia de la $\mathrm{TN}$ es cada vez más prominente $(4,7,10)$. El objetivo de este artículo fue revisar la evidencia actual disponible sobre la importancia clínica de la evaluación ecográfica de la TN fetal.

FISIOPATOLOGÍA DEL AUMENTO DE LA TRANSLUCENCIA NUCAL FETAL

El aumento del valor de la TN fetal está asociado con varias malformaciones y anomalías fetales, por lo que su etiología y fisiopatología son diversas (11). Los genes tanto de las proteínas de la matriz, como de los diferentes colágenos, están ubicados en los cromosomas 13, 18 y 21 . En fetos con trisomías de alguno de estos cromosomas, la producción de colágeno aumenta debido a las señales de origen genético. Este aumento de los componentes provoca aumento de la cantidad de líquidos y de elasticidad de los tejidos, apareciendo como aumento de volumen de la nuca fetal.

Otro de los mecanismos involucrados en el aumento de la TN fetal es la insuficiencia cardiaca fetal en etapas tempranas del embarazo (12). Los fetos con valores de TN más elevados tienen mayor riesgo de $\mathrm{CC}$, generalmente debidas a anomalías cardiacas estructurales (13-15). Sin embargo, no todos los fetos con CC presentan aumento de la TN. Además, las alteraciones hemodinámicas fetales no se manifiestan de forma uniforme como aumento de la $\mathrm{TN}$ en todos los fetos $(16,17)$.

La tercera hipótesis propone que la TN aumenta como consecuencia del retraso o deterioro del desarrollo del sistema linfático fetal. En fetos normales, en el sistema linfático, el saco linfático yugular se diferencia de las células endoteliales vasculares, convirtiéndose en vasos linfáticos, y el líquido que contiene fluye hacia el torrente sanguíneo. Este proceso, normalmente, debe completarse alrededor de la décima semana de embarazo (18). Sin embargo, algunas evidencias 


\section{IMPORTANCIA CLÍNICA DE LA EVALUACIÓN ECOGRÁFICA \\ DE LA TRANSLUCENCIA NUCAL FETAL}

demuestran que, en fetos con aumento del valor de la TN, el saco linfático del cuello está dilatado y la formación del sistema linfático normal está retrasada.

Además, algunas infecciones fetales (como toxoplasmosis, rubéola, citomegalovirus, herpes virus y parvovirus B19) producen anemia y congestión de las venas cefálicas fetales (19). Por otro lado, existen otras causas de aumento del valor de la TN fetal como compresión mediastínica y bandas amnióticas (19).

\section{MEDICIÓN DE LA TRANSLUCENCIA NUCAL FETAL}

Si la técnica de medición de la TN fetal es incorrecta, pueden existir casos en los que algunas malformaciones fetales pasan desapercibidas (falso negativo) o existe la posibilidad que se hagan pruebas invasivas innecesarias (falso positivo). Por tanto, el ecografista debe conocer de forma precisa, el método para su medición
(Figura 2). Debido a que el resultado de la medición puede variar de manera específica, los pequeños errores en el posicionamiento del transductor, pueden llevar a valores erróneos; especialmente debido a que la medición es realizada a intervalos de $0,1 \mathrm{~mm}$ (Tabla 1 y Figura 3) $(20,21)$.

\section{CRITERIOS DIAGNÓSTICOS PARA ESTABLECER EL AUMENTO DE LA TRANSLUCENCIA NUCAL FETAL}

En los primeros estudios, el criterio aceptado para considerar el aumento de la TN era de $3 \mathrm{~mm}$ (3). Sin embargo, a medida que aumenta la longitud céfalocaudal (LCC) fetal, también aumenta el grosor de la TN (22). Según algunas investigaciones, en un feto normal, para una LCC fetal de $45 \mathrm{~mm}$, la mediana y el percentil 95 son de $1,2 \mathrm{~mm}$ y $2,1 \mathrm{~mm}$, respectivamente; y para una LCC de $84 \mathrm{~mm}$ son $1,9 \mathrm{~mm}$ y $2,7 \mathrm{~mm}$, respectivamente $(6,23)$. Por tanto,

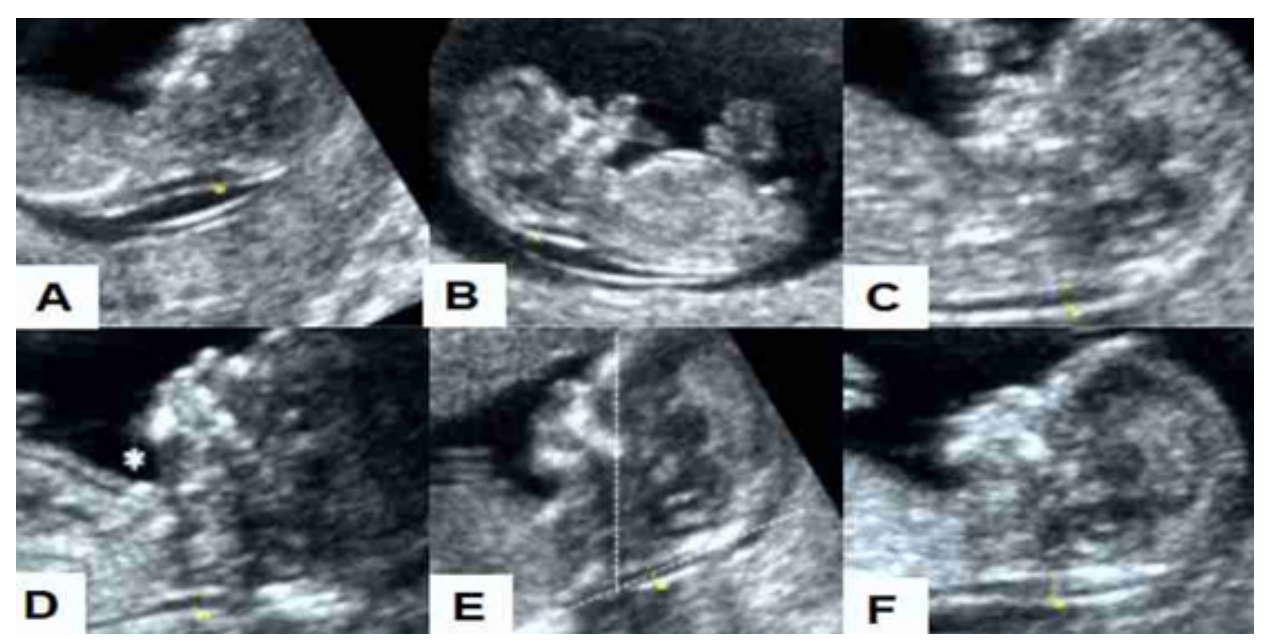

Figura 2. Ejemplos de mediciones de la translucencia nucal fetal. (A) Plano medio sagital inadecuado.

(B) Orientación medio sagital adecuada pero inadecuado para la medición. (C) Orientación medio sagital adecuada. Sin embargo, no existe espacio ecolúcido entre mentón y tórax fetal, lo cual puede llevar a subestimación del valor. (D) Cuello fetal en posición neutra con espacio ecolúcido $(*)$ entre mentón y tórax, pero la piel fetal no puede diferenciarse del amnios. (E) Plano medio sagital inadecuado, el eje no es perpendicular al eje mayor de la translucencia nucal, por lo que es posible que sea poco confiable la medición.

(F) Orientación medio sagital adecuada, aumento adecuado y cuello fetal neutral. 
Tabla 1. Enfoque estandarizado para la medición de la translucencia nucal fetal

PRERREQUISITOS

- El ecografista debe haber recibido capacitación formal y estar acreditado para la medición de la translucencia nucal.

- El paciente debe haber suministrado su consentimiento informado para la determinación.

- Edad gestacional entre 10-13 semanas o longitud céfalo-caudal entre 38-84 mm.

- La ecografía puede ser abdominal o transvaginal, con un equipo con capacidad cineloop. Los calibradores deben ser capaces de medir variaciones de $0,1 \mathrm{~mm}$.

- Es necesario disponer de tiempo suficiente para la determinación (aproximadamente 20 minutos)

IMAGEN Y MEDICIÓN DE LA TRANSLUCENCIA NUCAL FETAL

- La imagen debe ajustarse para que la cabeza, cuello y tórax fetal ocupen al menos el 75 \% de la pantalla, con una resolución ajustada.

- El perfil fetal debe observarse en su plano medio sagital y el ángulo de insonación debe estar perpendicular a la translucencia nucal.

- El cuello fetal debe estar en posición neutral, ni flexionado (mentón sobre el tórax pecho) ni extendido.

- Debe distinguirse el borde dorsal de la piel fetal del amnios subyacente.

- Es obligatorio medir el espesor máximo de la translucencia nucal.

Los calibradores deben colocarse de manera que las barras transversales estén colocadas dentro de la línea ecogénica en el borde más

- interno, pero no dentro el espacio ecolúcido, y alineados perpendicularmente al eje longitudinal del cuerpo fetal. Tampoco deben ser visibles dentro del espacio ecolúcido.

\section{INFORME DE LA TRANSLUCENCIA NUCAL}

- Deben realizarse varias mediciones. Para la evaluación del riesgo, se puede utilizar la mayor de al menos 3 mediciones de buena calidad.

Cuando el cordón umbilical produzca algún artefacto que lleve a diferencias en el valor de alguno de los lados, se puede reportar la - medida más pequeña o el valor promedio

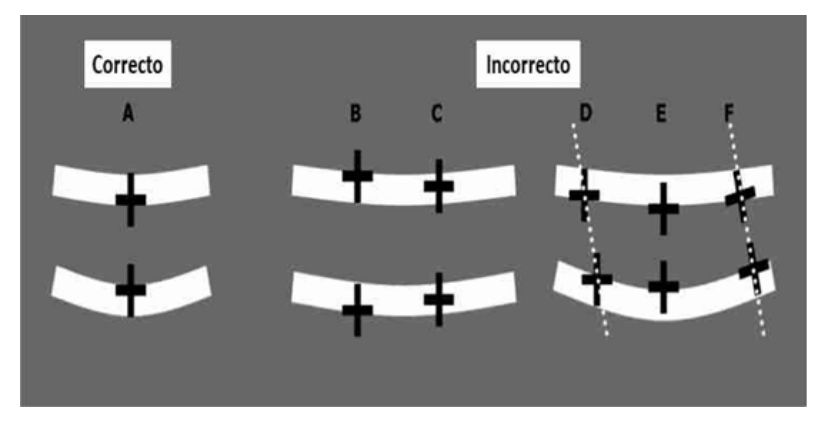

Figura 3. Medición de la translucencia nucal fetal. Colocación adecuada de calibradores. (A): Estos deben colocarse en el borde más interno de las líneas ecogénicas, de modo que las barras transversales coincidan con estas. Colocación incorrecta de los calibradores con relación a las líneas ecogénicas:

(B) Colocados en el borde más externo.

(C) Colocados en el centro. (D) No están alineados perpendicularmente al eje mayor de la translucencia. (E) Las barras transversales son visibles dentro del espacio ecolúcido. (F) Relativamente bien alineados al eje mayor de la translucencia nucal, pero no se mide el valor máximo. en lugar de utilizar un valor fijo predeterminado, es recomendable establecer el percentil 95 sobre la base de la LCC. Una investigación demostró que cuando se utilizó un valor fijo predeterminado para establecer la anormalidad de la TN fetal, la sensibilidad y la tasa de falsos positivos para el diagnóstico de aneuploidía fetal, fueron mayores, comparadas con los valores obtenidos cuando fueron utilizados los valores basales del percentil 95 de la LCC (24).

Durante el primer trimestre del embarazo, las concentraciones séricas de proteína plasmática-A asociada al embarazo (PAPP-A) y $\beta$-gonadotropina coriónica humana libre ( $\beta$-hCG) son marcadores bioquímicos para la detección de aneuploidías (25). Sus valores son convertidos a múltiplos de la mediana $(\mathrm{MoM})$ de las concentraciones séricas promedio de embarazadas normales a la semana gestacional correspondiente, y son utilizados como exámenes de detección complejos. La medición de la TN fetal también utiliza valores de MoM, pero 


\section{IMPORTANCIA CLÍNICA DE LA EVALUACIÓN ECOGRÁFICA \\ DE LA TRANSLUCENCIA NUCAL FETAL}

existe controversia sobre el uso de valores obtenidos de la población general o individualizados de cada institución o servicio. Un estudio demostró que, comparado con el uso de valores obtenidos de la población general, utilizar MoM de la $\mathrm{TN}$ de cada evaluador, puede aumentar la tasa de detección del $\mathrm{SdD}$ en cerca de un $5 \%$ (8). Otra investigación sugirió la posibilidad de utilizar la diferencia (delta) del valor de la mediana de embarazadas normales por edad de gestación, ya que es más exacto que calcular el MoM. Los autores encontraron que el uso de MoM podría sobrestimar el riesgo de $\mathrm{SdD}$ a las 11 semanas, y subestimar este riesgo, a las 13 semanas de gestación (20).

La mayoría de los estudios demuestran que, en el primer trimestre, en embarazos normales, el aumento de la TN fetal desaparece de forma espontánea, después de las 14 semanas; pero en casos de aumento de volumen de la nuca o en fetos con higroma quístico, pueden persistir algunos engrosamientos (figura 4) (26). En la evaluación ecográfica es posible observar estas lesiones que persisten, como quistes anecoicos del tejido blando de la nuca fetal, con tabiques o trabéculas en su interior, limitados a la nuca, a lo largo de la espalda fetal o en todo el cuerpo. También puede presentarse como edema subcutáneo. Las lesiones linfáticas fetales están asociadas con anomalías cromosómicas fetales, particularmente SdD y síndrome de Turner (27).

El saco linfático, que es observable entre las 10-14 semanas de embarazo, puede considerarse como parte de una forma extrema del aumento de la TN fetal. Existe controversia sobre si debe considerarse como una condición diferente. Estudios iniciales no tomaban en cuenta la presencia de sacos linfoides (6). No obstante, un estudio posterior demostró que este saco se extendía a toda la LCC, y, si existía un septo en su interior, podía ser definido como un saco linfático; y si estaba presente, su interpretación debía ser diferente (7). Otro estudio sobre la presencia de tabiques dentro de los quistes en la nuca fetal demostró que solo el grosor de la TN fetal, y no su longitud, era predictor de aneuploidías (28). Aunque aparentemente no es necesario considerar la presencia del saco linfático como una prueba de detección de aneuploidías, es necesario obtener conclusiones definitivas.

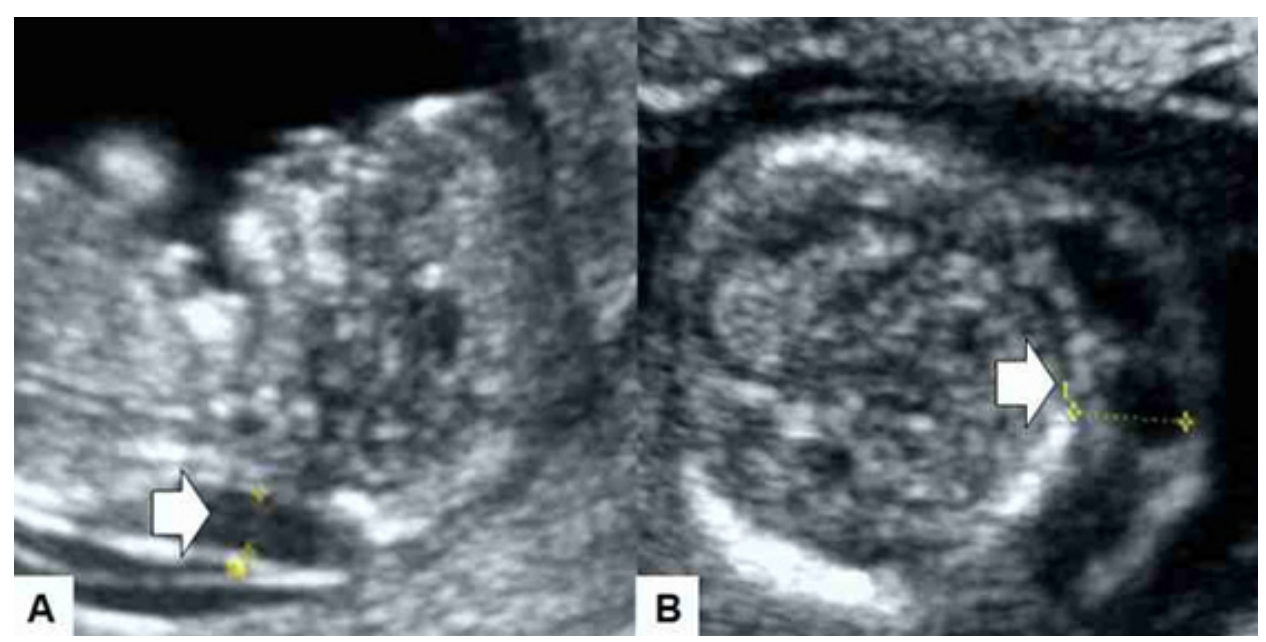

Figura 4. (A) La flecha señala translucencia nucal aumentada en feto de 13 semanas de gestación. (B) La flecha señala los tabiques y trabéculas visibles dentro del higroma quístico en un feto de 13 semanas de gestación. 
AUMENTO DE LA TRANSLUCENCIA NUCAL FETAL Y ANEUPLOIDÍAS FETALES

La asociación entre el aumento de la TNy el diagnóstico de aneuploidías fetales es reconocida $(3,6)$. Por tanto, esta medición es utilizada como herramienta de detección de estas alteraciones cromosómicas. Aproximadamente $75 \%-80 \%$ de las aneuploidías fetales, incluido el $\mathrm{SdD}$, pueden diagnosticarse, con una tasa de falsos positivos cercana al $5 \%(20,29)$. El riesgo de aneuploidías fetales aumenta en relación directa con el aumento del grosor de la TN. Cuando el grosor es inferior al percentil 95, la posibilidad de aneuploidía es de 0,2\%. Cuando el valor se encuentra entre los percentiles 95-99, la posibilidad aumenta hasta 3,77\%. Cuando supera el percentil 99, la posibilidad de aneuploidías asociadas varía entre $21 \%$ - $65 \%$; dependiendo del valor de la TN y la edad de gestación (5).

Las embarazadas con fetos diagnosticados de SdD, tienen concentraciones séricas de $\beta$-hCG libre más elevadas (por encima de $2 \mathrm{MoM}$ ) y concentraciones de PAPP-A menores (inferiores a 0,5 MoM) $(30,31)$. Al momento de la determinación de las pruebas de detección bioquímica, es necesario considerar la edad de gestación, ya que a medida que avanza el embarazo, disminuye la diferencia con respecto a los valores normales de PAPP-A, y aumenta la diferencia con la $\beta$-hCG (32). Por tanto, durante el primer trimestre del embarazo, comparado con las pruebas realizadas en forma aislada, al agregar la determinación de la TN fetal a estas pruebas, aumenta la precisión.

Durante el primer trimestre del embarazo, los estudios sobre detección del $\mathrm{SdD}$ han demostrado que la evaluación de la TN fetal combinada con las pruebas bioquímicas, con tasa de falsos positivos de $5 \%$, tiene un porcentaje de detección de 78,7\% (intervalo de confianza del $95 \%, 66,3 \%-88,1 \%$ ) (9). Un estudio que utilizó pruebas bioquímicas durante el segundo trimestre, mostró que, con una tasa de falsos positivos del $5 \%$, la detección de SdD fue cercana al $87 \%$. La combinación de las pruebas ecográficas y bioquímicas fue superior a la prueba cuádruple (83\%) y a la prueba triple (73\%) (4). Finalmente, otra investigación mostró que para la detección de $\mathrm{SdD}$, las tasas de detección para la TN fetal combinada con pruebas bioquímicas, fueron $87 \%$, $85 \%$ y $82 \%$, a las 11,12 y 13 semanas de embarazo, respectivamente. La frecuencia de falsos positivos fue de $5 \%$. A la semana 11 de embarazo, en forma específica, la prueba de detección combinada (TN más pruebas bioquímicas) resultó ser superior a la prueba cuádruple (tasa de detección del $83 \%$, con frecuencia de falsos positivos del $5 \%$ ) (7).

Durante el primer trimestre del embarazo, la prueba de detección combinada con marcadores bioquímicos, también puede establecer el riesgo de otras anomalías cromosómicas diferentes al SdD. Comparados con fetos normales, las concentraciones séricas de $\beta$-hCG y PAPP-A de fetos con trisomía 18 (síndrome de Edward) y trisomía 13 (síndrome de Patau) son más bajas. En fetos con triploidías de cromosomas paternos, la $\beta$-hCG libre aumenta y la PAPP-A disminuye ligeramente; mientras que aquellas cromosomopatías de origen materno, tanto la $\beta$-hCG como la PAPP-A disminuyen en forma significativa (20). Durante el primer trimestre del embarazo, los resultados de utilizar estos marcadores, demuestran que la tasa de detección de trisomía 18 , osciló entre $2 \%$ - $91 \%$. No obstante, todavía no existen suficientes estudios con un número adecuado de pacientes (33).

En el primer trimestre del embarazo, además, del aumento de la TN fetal, existen otros indicadores ecográficos que podrían sugerir el diagnóstico de $\mathrm{SdD}$, incluyendo: regurgitación tricúspidea, onda doppler del flujo sanguíneo anormal del conducto venoso y características del hueso nasal. 


\section{IMPORTANCIA CLÍNICA DE LA EVALUACIÓN ECOGRÁFICA \\ DE LA TRANSLUCENCIA NUCAL FETAL}

Entre las 11-14 semanas de gestación, la regurgitación tricúspidea ocurre aproximadamente en $5 \%$ de los fetos normales, mientras que $70 \%$ de los fetos con $\mathrm{SdD}$ presentan esta alteración $(34,35)$. Existe evidencia que demuestra que, durante el primer trimestre del embarazo, cuando se incluyó la regurgitación de la válvula tricúspidea en la evaluación ecográfica, la tasa de detección del SdD fue superior al 90 \% (36).

Un estudio que evaluó fetos con anomalías fetales, observó reversión o pérdida de la onda venosa $\mathrm{A}$ en $91 \%$ de los casos con cromosomopatías y solo en $3 \%$ de los fetos normales (37). Otros estudios informaron que, en el primer trimestre, la tasa de detección de cromosomopatías por alteraciones del doppler venoso, con una tasa de falsos positivos de $2 \%-21 \%$, es del $59 \%$ - $93 \%$ (38). Sin embargo, la combinación de aumento de la TN y hallazgos del doppler venoso, no mejoró la capacidad de detección de aneuploidías fetales, pero si aumentó la especificidad y la razón predictiva positiva $(39,40)$.

En fetos normales, solo $1 \%-2 \%$ tienen alteraciones de los huesos nasales, mientras que $60 \%-70 \%$ de los fetos con SdD presentan estas alteraciones. En la evaluación ecográfica a las 11-13 semanas de gestación, cuando se identifican alteraciones de los huesos nasales, con una tasa de falsos positivos de $1,4 \%$, la frecuencia de diagnóstico del $\mathrm{SdD}$ es de $69 \%$ (41). Al incluir este hallazgo, con una tasa de falsos positivos cercana a $2,5 \%$, la tasa diagnóstica puede llegar hasta $90 \%(42,43)$.

Lamentablemente, la búsqueda de estos hallazgos ecográficos necesita de mayor tiempo y experiencia del evaluador, al igual que una máquina de ecografía sofisticada. También, existe alto riesgo de errores debido a mediciones o interpretaciones inexactas (44). Además, cuando son realizadas en la población general y son utilizadas como pruebas únicas para la detección de $\mathrm{SdD}$, pueden existir inconvenientes; ya que la sensibilidad es baja (menos de $15 \%$ ) (1,
20). Sin embargo, en embarazadas de alto riesgo, la combinación de estos hallazgos con otros marcadores, puede ser útil en función de corrección de la probabilidad de detección y la tasa de falsos positivos $(42,45)$.

\section{AUMENTO DE LA TRANSLUCENCIA NUCAL FETAL EN FETOS SIN ANEUPLOIDÍAS}

Si durante el primer trimestre del embarazo, está aumentado el grosor de la TN fetal, aumenta el riesgo de aborto y muerte perinatal, junto con el aumento del riesgo de anomalías congénitas importantes (incluidas CC), incluso si no existen cromosomopatías fetales (5, 46 - 49).

\section{Malformaciones congénitas}

Durante el primer trimestre del embarazo, el aumento del grosor de la TN fetal está fuertemente asociado con anomalías estructurales fetales $\mathrm{y}$, al igual que con las aneuploidías, la frecuencia de anomalías congénitas importantes aumenta en relación directa con el aumento de la $\operatorname{TN}(5,13,15,50)$. La frecuencia de anomalías congénitas mayores por debajo del percentil 95 es de $1,6 \%$, mientras que en el percentil $95-99$ es de $2,5 \%$. Para valores de 3,5-4,4 mm es de $10 \%$, de 4,5-5,4 mm es de $18,5 \%$, para valores de 5,5-6,4 mm es de $24,2 \%$, y para valores superiores a $6,5 \mathrm{~mm}$, es de 46,2 \% (5).

Las CC son la más comúnmente asociadas al aumento del valor de la TN. Entre las 11-14 semanas de embarazo, aproximadamente $83 \%$ de los fetos con cardiopatías detectadas por ecocardiografía, presentan aumento de la TN (51). Sin embargo, existen algunos informes de aumento de la TN en fetos sin cromosomopatías. En estos casos la incidencia de cardiopatías mayores es de $3 \%-5 \%$, lo cual es significativamente superior a la población general $(5,52-54)$.

Otro hallazgo importante es que la incidencia de CC aumenta a medida que aumenta el grosor de la TN (29, 
$53,55,56)$. La frecuencia de anomalías de acuerdo a los valores de TN fetal menor del percentil 95 fue de $1,6 \% ; 3 \%$ en el percentil $95-99 ; 7 \%$ entre 3,5-4,4 $\mathrm{mm} ; 18,5 \%$ entre $4,5-5,4 \mathrm{~mm} ; 20 \%$ entre 5,5-6,4 mm y $30 \%$ por encima de $6,5 \mathrm{~mm}$ (5). Cuando el valor de la TN supera los $3 \mathrm{~mm}$, la prevalencia de CC puede superar $10 \%(29)$.

Varias investigaciones han evaluado el papel de la TN fetal como única prueba de predicción de CC. Un análisis demostró que la tasa de predicción, fue solo del $31 \%$ - $37 \%$ (57). Estos resultados demuestran que, debido a su baja sensibilidad, el aumento de los valores de TN no es útil para la predicción (58). Además, tampoco tiene la capacidad de predecir CC específicas (59). Por otro lado, cuando el aumento del valor de la TN está asociado a pérdida o inversión de la onda A en la ecografía doppler venosa, el riesgo de CC aumenta aún más $(60,61)$. Por tanto, en estos fetos, la suma de la evaluación doppler venosa, puede mejorar la precisión de la predicción de CC (62).

En los fetos con aumento de la TN, además de las CC, existe otra amplia variedad de anomalías estructurales que pueden diagnosticarse. Las malformaciones más importantes incluyen hernia diafragmática, exoftalmos, anomalía del tallo corporal y megavejiga $(5,29,63)$.

\section{Alteraciones del desarrollo infantil}

No existen suficientes estudios de seguimiento a largo plazo de niños con TN anormal durante el primer trimestre, sin malformaciones estructurales ni cromosomopatías. Investigaciones que utilizaron cuestionarios o pruebas clínicas informaron que la incidencia de alteraciones del desarrollo, fue menor del $3 \%$ (5). Sin embargo, comparado con el grupo de control, el número de sujetos en estos estudios, fue muy pequeño. Los resultados de otro estudio prospectivo en el que la TN fetal estaba por encima del percentil 99, no indicaron diferencias significativas en la frecuencia de alteraciones del desarrollo hasta 2 años después del nacimiento (64).

\section{Muerte fetal intrauterina}

El aumento de la TN en fetos normales sin cromosomopatías está asociado con aumento en el riesgo de muerte fetal intrauterina y este riesgo aumenta en forma directamente proporcional al aumento del grosor. Para los fetos en el percentil 99, la tasa de mortalidad es cercana a 1,3\%, la cual es similar a la de los fetos normales con valores por debajo del percentil $95(65,66)$. Un estudio con fetos normales con valores de TN superior a 3,5 mm demostró que la tasa de abortos o mortinatos fue de 5,1\%. El $88 \%$ de los eventos ocurrieron antes de las 20 semanas gestación. Aquellos fetos que superaron las 20 semanas y no presentaron alteraciones estructurales, presentaron una tasa de muerte perinatal de 2,2\% (66). No obstante, en los fetos con aumento de la TN que sobrevivieron, si estaba acompañada de anomalías estructurales, presentaron una resultante perinatal peor. Los casos de muerte perinatal y discapacidad que necesitaron tratamiento médico o quirúrgico, alcanzaron $18 \%$ (65). Por tanto, incluso en ausencia de cromosomopatías, si la TN fetal está aumentada, es necesario confirmar la presencia de anomalías estructurales mediante evaluaciones ecográficas específicas durante el embarazo. Asimismo, si existe evidencia de aumento del grosor de la $\mathrm{TN}$, es particularmente importante establecer el riesgo de aborto espontáneo y muerte perinatal.

\section{TRANSLUCENCIA NUCAL EN EMBARAZOS MÚLTIPLES}

La TN es útil como prueba de detección de anomalías fetales, incluso en los embarazos múltiples (EM). Durante el primer trimestre, en embarazos gemelares (EG), el uso de la medición sola o junto a pruebas de 


\section{IMPORTANCIA CLÍNICA DE LA EVALUACIÓN ECOGRÁFICA \\ DE LA TRANSLUCENCIA NUCAL FETAL}

detección compleja, con una tasa de falsos positivos de $5 \%$ - $7 \%$, puede diagnosticar $75 \%$ - $88 \%$ de los fetos con SdD (67 - 69). No obstante, comparado con el embarazo simple, el cribado de aneuploidías en EM necesita mayor atención debido a que el grosor de la TN de cada gemelo, frecuentemente no coincide. Existen métodos para calcular el riesgo individual para cada feto (estimación del riesgo específico del feto) y estimación del riesgo específico del embarazo (combinando los riesgos de ambos fetos). Sin embargo, en las pacientes con EM, dado que no existen diferencias en las concentraciones de marcadores bioquímicos, cuando se realiza una prueba de detección compleja, es imposible calcular el riesgo para cada feto. Esto significa que es imposible calcular el riesgo de cada feto en forma particular, pero si se calcula el riesgo del EG en sí, lo que se ha denominado "pseudorriesgo" (70).

En EM, la corionicidad es un factor importante para la determinación del riesgo de aneuploidías (71). En embarazos monocigóticos, el riesgo de $\mathrm{SdD}$ es el mismo para ambos fetos, mientras que, en gemelos dicigóticos, el riesgo es independiente entre ellos. En vista a lo anterior expuesto, uno de los gemelos heterocigotos puede ser diagnosticado con $\mathrm{SdD}$ y el otro gemelo puede carecer de la condición (70-72).

En gemelos monocoriónicos (GMC), aunque presenten valores similares de translucencia fetal, el riesgo de aneuploidía puede ser diferente. Un estudio que comparó el grosor de la TN entre GMC, demostró que la tasa de sensibilidad para la detección de SdD fue de 5,1\% para el valor mayor; 45,9\% para el valor menor y 4,2 \% para el valor promedio (73). Estos resultados sugieren que, para el diagnóstico de $\mathrm{SdD}$, el valor promedio de ambos gemelos es más efectivo. Por tanto, en GMC, el riesgo debe calcularse utilizando el promedio de los valores de MoM de la TN de ambos fetos (74). Por otra parte, existe evidencia que, los GMC, comparados con los gemelos bicoriónicos (GBC) o fetos de embarazos simples, tienen mayor riesgo de presentar valores de $\mathrm{TN}$ iguales o superiores al percentil 95 (69). Algunos investigadores sugieren que esto puede ser un signo temprano del síndrome de transfusión feto-feto (75). Por lo tanto, en los GMC, comparados con los fetos de embarazos simples, la tasa de falsos positivos del grosor de la TN, es más alta (71).

En los GBC, el riesgo de aneuploidía de los fetos es diferente y las mediciones de TN no siempre están asociadas con el riesgo global. Para estos casos, debe realizarse la medición de la $\mathrm{TN}$ de cada gemelo, de acuerdo con la LCC de forma individual, el riesgo debe ser analizado por separado; y luego, establecer una conclusión final. En la práctica clínica cotidiana, rara vez se realiza solo la evaluación de uno de los fetos. Por tanto, es deseable y fundamental calcular el riesgo individual para cada uno de los fetos $(70,74)$.

En los EM, las concentraciones de PAPP-A y $\beta$-hCG, comparadas con los embarazos simples, son aproximadamente 1,86 - 2,10 veces más altas. Además, no existen diferencias entre los tipos de corionicidad (69). En los EG, las mediciones de PAPP-A para poder establecer el riesgo, deben ajustarse; restando el aumento del valor asociado $(70,74)$. En los GBC, debido a que el riesgo de aneuploidía entre los fetos es diferente, el cálculo del riesgo es más complejo; pero la concentración de marcadores bioquímicos es la misma para ambos. En particular, en caso que uno de los fetos tenga alguna cromosomopatía, cuando el otro feto es normal; los cambios en las concentraciones de PAPP-A y $\beta$-hCG libre, pueden afectar las conclusiones. Para estos casos es deseable calcular el riesgo del embarazo, en lugar del riesgo de cada feto en forma individual $(70,71,74)$.

En embarazos de tres o más fetos incluso, existen informes de mediciones de la TN fetal, y no existen diferencias con la técnica de medición utilizada en embarazos simples $(76,77)$. Sin embargo, en este tipo de casos, aún no existen conclusiones definitivas. 
MANEJO DE LOS CASOS CON AUMENTO DE LA TRANSLUCENCIA NUCAL FETAL

El manejo de los fetos con aumento de la TN durante el primer trimestre del embarazo, se muestra en la Figura $5(4,5)$. Para aquellos casos en los cuales la TN es inferior al percentil 95 (normal), la probabilidad de malformaciones congénitas mayores es de alrededor del $3 \%$. Si el valor del grosor está entre los percentiles 95-99, varios factores como edad de la embarazada, hallazgos ecográficos y concentración sérica de PAPP-A y $\beta$-hCG libre, pueden ser útiles para establecer el diagnóstico de aneuploidías. En estos casos, la posibilidad de obtener un feto sin cromosomopatías, es de alrededor del $7 \%$. Si el valor supera el percentil 99, o los 3,5 mm, la posibilidad de cromosomopatías es elevada, por lo que es necesario realizar pruebas invasivas para estudio del genotipo fetal.
Durante el primer trimestre del embarazo, por medio de la evaluación ecográfica precisa, es posible diagnosticar varias anomalías estructurales fetales importantes. La evaluación ecográfica seriada puede ser de ayuda en aquellos casos en que las pruebas de detección no suministren información suficiente y el estudio genético del feto sea normal. En la mayoría de los fetos, el aumento en los valores de TN, desaparece espontáneamente después de las 14 semanas de embarazo; pero en algunos casos puede persistir como aumento de volumen de la zona de la nuca o presencia de un saco linfático.

Si no existen anomalías estructurales y la TN desaparece, la posibilidad de malformaciones mayores está por debajo del $5 \%$ y el seguimiento ecográfico debe realizarse nuevamente a las 20-22 semanas de embarazo. El objetivo de esta evaluación es detectar

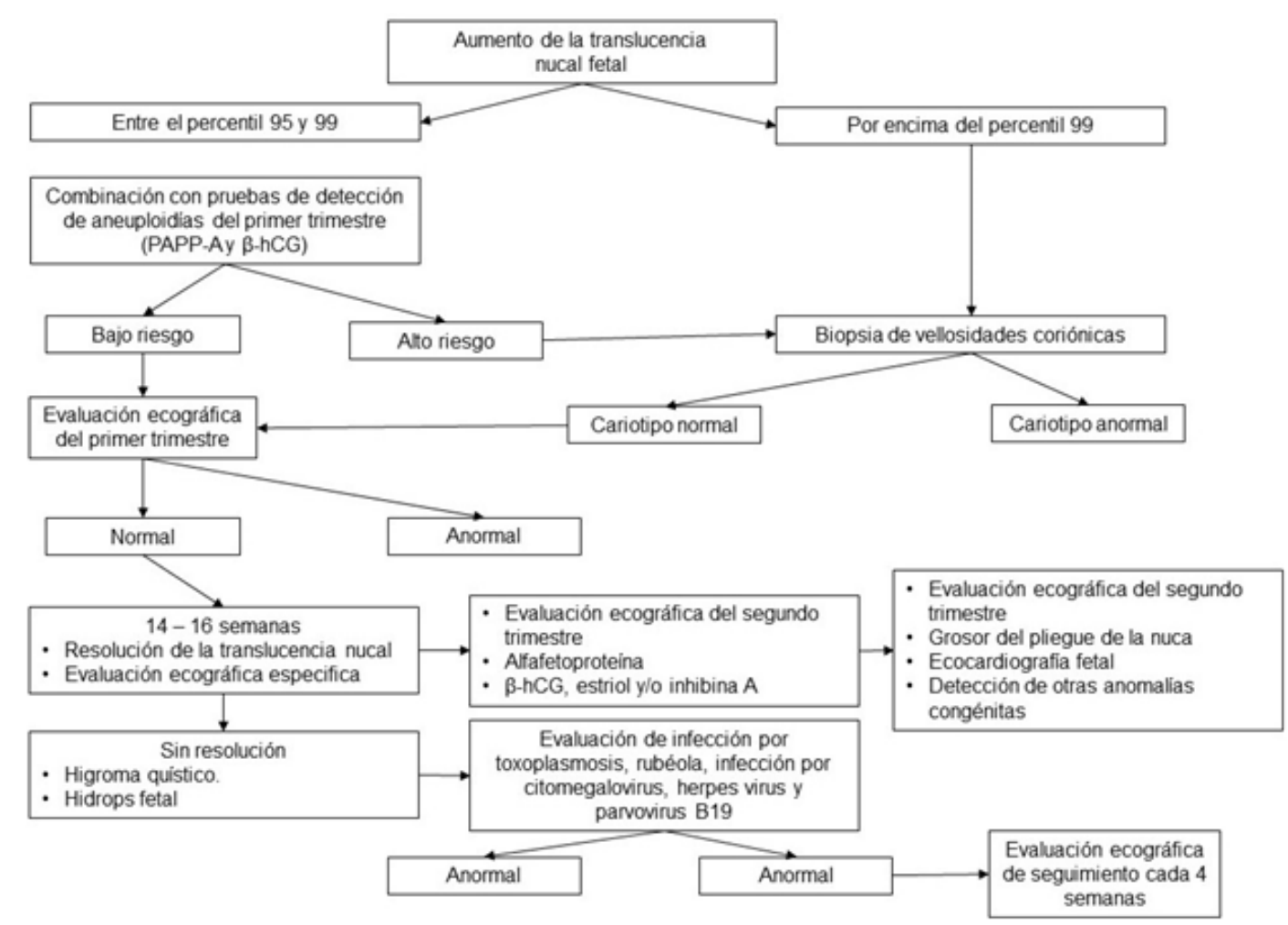

Figura 5. Algoritmo del manejo de los fetos con aumento de la translucencia nucal durante el primer trimestre del embarazo. 


\section{IMPORTANCIA CLÍNICA DE LA EVALUACIÓN ECOGRÁFICA \\ DE LA TRANSLUCENCIA NUCAL FETAL}

posibles anomalías que no fueron detectadas en la evaluación del primer trimestre $\mathrm{y}$, en particular, para establecer la presencia de CC fetales.

Si a las 14-16 semanas de embarazo, se observa aumento de la TN, existe evidencia de hidropesía fetal o presencia de saco linfático, es necesario considerar la posibilidad de infección congénita o enfermedad cromosómica fetal. En estos casos es necesario realizar pruebas para establecer la posibilidad de infección por toxoplasma, rubéola, citomegalovirus, herpes virus y parvovirus B19. Además, para evaluar la evolución del grosor de la zona de la nuca fetal, es necesario realizar evaluaciones ecográficas cada 4 semanas.

Si el aumento del grosor del TN fetal aparece sin causa específica, incluso entre las 20-22 semanas de gestación, es necesario considerar la probabilidad de hidropesía fetal o aneuploidías; que representan aproximadamente el $10 \%$ de los casos de aumento de volumen de la región de la nuca (48).

\section{CONCLUSIONES}

Durante el primer trimestre del embarazo:

- El aumento de la TN fetal tiene fuerte asociación con cromosomopatías y anomalías estructurales fetales, incluido el SdD.

- La combinación con otras pruebas como PAPP-A y $\beta$-hCG libre, puede mejorar la precisión diagnóstica.

- Es posible realizar una detección más precisa de estas alteraciones añadiendo hallazgos adicionales como presencia de alteraciones de los huesos nasales, formas anormales de onda de flujo sanguíneo doppler venosas e insuficiencia de la válvula tricúspide.

- Los fetos con aumento de grosor de la TN y sin cromosomopatías, tienen peor resultante perinatal.
Durante el segundo trimestre del embarazo:

- Los fetos diagnosticados con aumento de la TN durante el primer trimestre deben ser monitorizados regularmente con evaluaciones ecográficas específicas.

\section{Sin conflictos de interés.}

\section{REFERENCIAS}

1. Nemescu D, Bratie A, Mihaila A, Navolan D, Tanase A. First trimester combined screening for fetal aneuploidies enhanced with additional ultrasound markers: an 8-year prospective study. Ginekol Pol. 2018; 89(4):205-10. doi: 10.5603/GP.a2018.0035.

2. Nisbet D, Robertson A, Mannil B, Pincham V, Mclennan A. Quality management of nuchal translucency ultrasound measurement in Australia. Aust N Z J Obstet Gynaecol. 2019; 59(1):54-58. doi: 10.1111/ajo.12792.

3. Nicolaides KH, Azar G, Byrne D, Mansur C, Marks K. Fetal nuchal translucency: ultrasound screening for chromosomal defects in first trimester of pregnancy. BMJ. 1992; 304(6831):867-869. doi: 10.1136/ bmj.304.6831.867.

4. Alldred SK, Takwoingi Y, Guo B, Pennant M, Deeks JJ, Neilson JP, et al. First trimester ultrasound tests alone or in combination with first trimester serum tests for Down's syndrome screening. Cochrane Database Syst Rev. 2017; 3(3):CD012600. doi: 10.1002/14651858. CD012600.

5. Souka AP, Von Kaisenberg CS, Hyett JA, Sonek JD, Nicolaides KH. Increased nuchal translucency with normal karyotype. Am J Obstet Gynecol. 2005; 192(4):1005-1021. doi: 10.1016/j.ajog.2004.12.093.

6. Huang T, Gibbons C, Rashid S, Priston MK, Bedford HM, Mak-Tam E, et al. Prenatal screening for trisomy 21: a comparative performance and cost analysis of different screening strategies. BMC Pregnancy Childbirth. 2020; 20(1):713. doi: 10.1186/s12884-02003394-w.

7. Luo W, He B, Han D, Yuan L, Chen X, Pang L, et al. A retrospective analysis of different contingent screening models for fetal down syndrome in Southwestern China. Sci Rep. 2020; 10(1):9457. doi: 10.1038/ s41598-020-66320-2.

8. Wald NJ, Rodeck C, Hackshaw AK, Walters J, Chitty L, Mackinson AM. First and second trimester 
antenatal screening for Down's syndrome: the results of the Serum, Urine and Ultrasound Screening Study (SURUSS). J Med Screen. 2003; 10(2):56-104. doi: 10.1258/096914103321824133.

9. Berger VK, Norton ME, Sparks TN, Flessel M, Baer RJ, Currier RJ. The utility of nuchal translucency ultrasound in identifying rare chromosomal abnormalities not detectable by cell-free DNA screening. Prenat Diagn. 2020; 40(2):185-190. doi: 10.1002/pd.5583.

10. Cedeño-Rincón R. La trisomía 21: 50 años después de Lejeune. Gac Med Caracas [Internet]. 2010 [consultado diciembre 2020]; 118(3):203-211. Disponible en: http://190.169.30.98/ojs/index.php/rev_gmc/article/ view/18139

11. Liao KW, Tsai MS, Chang CH, Chien LC, Mao IF, Tsai YA, et al. Do the Levels of Maternal Plasma Trace Elements Affect Fetal Nuchal Translucency Thickness? PLoS One. 2015; 10(9):e0138145. doi: 10.1371/ journal.pone.0138145.

12. Oh SY, Hong JS, Seol HJ, Hwang HS, Park HS, Kim $\mathrm{K}$, et al.; Korean Society of Ultrasound in Obstetrics and Gynecology Research Group. 2014 First-trimester ultrasound forum from the Korean Society of Ultrasound in Obstetrics and Gynecology. Obstet Gynecol Sci. 2015; 58(1):1-9. doi: 10.5468/ogs.2015.58.1.1.

13. Şahin Uysal N, Gülümser Ç, Yılmaz Çelik Z, Yanık FB. Increased nuchal translucency and pregnancy outcomes: experience of Başkent University Ankara Hospital. Turk J Obstet Gynecol. 2019; 16(2):100-106. doi: 10.4274/tjod.galenos.2019.51482.

14. Clur SA, Ottenkamp J, Bilardo CM. The nuchal translucency and the fetal heart: a literature review. Prenat Diagn. 2009; 29(8):739-748. doi: 10.1002/ pd.2281.

15. Clur SA, Oude Rengerink K, Mol BW, Ottenkamp J, Bilardo CM. Fetal cardiac function between 11 and 35 weeks' gestation and nuchal translucency thickness. Ultrasound Obstet Gynecol. 2011; 37(1):48-56. doi: 10.1002/uog.8807.

16. Geipel A, Gembruch U. [Screening performance of first trimester nuchal translucency, ductus venosus blood flow and tricuspid regurgitation for cardiac defects]. Z Geburtshilfe Neonatol. 2012; 216(4):157-161. German. doi: $10.1055 / \mathrm{s}-0032-1316345$.

17. Salman Guraya S. The associations of nuchal translucency and fetal abnormalities; significance and implications. J Clin Diagn Res. 2013; 7(5):936-941. doi: $10.7860 / \mathrm{JCDR} / 2013 / 5888.2989$.
18. Eckmann-Scholz C, Salmassi A, Jonat W, Alkatout I. Distended jugular lymphatic sacs in fetuses with increased nuchal translucency: correlation with firsttrimester findings in aberrant karyotypes. J Matern Fetal Neonatal Med. 2014; 27(3):257-260. doi: 10.3109/14767058.2013.809416.

19. van de Pavert SA, Mebius RE. New insights into the development of lymphoid tissues. Nat Rev Immunol. 2010; 10(9):664-674. doi: 10.1038/nri2832.

20. Xu L, Huang H, Zheng L, He D, Lin N, Wang L, et al. Optimization of regional median equations of prenatal screening markers for trisomy 21 in a Chinese population. Medicine (Baltimore). 2018; 97(35):e12045. doi: 10.1097/MD.0000000000012045.

21. Merz E, Thode C, Alkier A, Eiben B, Hackelöer BJ, Hansmann M, et al. A new approach to calculating the risk of chromosomal abnormalities with first-trimester screening data. Ultraschall Med. 2008; 29(6):639-645. doi: 10.1055/s-2008-1027958.

22. Zoppi MA, Ibba RM, Floris M, Monni G. Fetal nuchal translucency screening in 12495 pregnancies in Sardinia. Ultrasound Obstet Gynecol. 2001; 18(6):649651. doi: 10.1046/j.0960-7692.2001.00583.x.

23. Kor-Anantakul O, Suntharasaj T, Suwanrath C, Chanprapaph P, Sirichotiyakul S, Ratanasiri T, et al. Distribution of normal nuchal translucency thickness: a multicenter study in Thailand. Gynecol Obstet Invest. 2011; 71(2):124-128. doi: 10.1159/000320754.

24. Kaul A, Radhakrishnan P. Performance of Common Down Syndrome Screening Methods Used in India with Construction of an Indian Normogram for Nuchal Translucency/Crown-Rump Length Measurements in 14,337 Subjects. J Obstet Gynaecol India. 2019; 69(Suppl 2):142-146. doi: 10.1007/s13224-018-11963.

25. Fernández H, Fernández H. Triple marcador bioquímico en el diagnóstico prenatal. Col Med Estado Táchira 2020; 11(2):3-5.

26. Spencer K, Bindra R, Nix AB, Heath V, Nicolaides KH. Delta-NT or NT MoM: which is the most appropriate method for calculating accurate patient-specific risks for trisomy 21 in the first trimester? Ultrasound Obstet Gynecol. 2003; 22(2):142-148. doi: 10.1002/uog. 186.

27. Kitazawa S, Mori K, Kondo T, Kitazawa R. Fetal nuchal cystic hygroma associated with aortic coarctation and trisomy 21: a case report. Cases J. 2009; 2:8280. doi: 10.4076/1757-1626-2-8280. 


\section{IMPORTANCIA CLIINICA DE LA EVALUACIÓN ECOGRÁFICA \\ DE LA TRANSLUCENCIA NUCAL FETAL}

28. Scholl J, Durfee SM, Russell MA, Heard AJ, Iyer C, Alammari R, et al. First-trimester cystic hygroma: relationship of nuchal translucency thickness and outcomes. Obstet Gynecol. 2012; 120(3):551-559. doi: 10.1097/AOG.0b013e318264f829.

29. Sulaiman B, Shehu CE, Panti AA, Saidu SA, Onankpa B, Ekele BA. Prevalence and outcome of increased nuchal translucency in usmanu danfodiyo university teaching hospital, Sokoto, Nigeria: A cohort study. Niger J Clin Pract. 2020; 23(6):864-869. doi: 10.4103/ njcp.njcp_191_19.

30. Huang S, Chang C, Cheng P, Hsiao C, Soong Y, Duan T. First-trimester combined screening is effective for the detection of unbalanced chromosomal translocations at 11 to 12 weeks of gestation. Reprod Sci. 2014; 21(5):594-600. doi: 10.1177/1933719113508818.

31. Spencer K, Cowans NJ, Avgidou K, Nicolaides KH. First-trimester ultrasound and biochemical markers of aneuploidy and the prediction of impending fetal death. Ultrasound Obstet Gynecol. 2006; 28(5):637-643. doi: 10.1002/uog.3809.

32. Spencer K, Crossley JA, Aitken DA, Nix AB, Dunstan FD, Williams $\mathrm{K}$. The effect of temporal variation in biochemical markers of trisomy 21 across the first and second trimesters of pregnancy on the estimation of individual patient-specific risks and detection rates for Down's syndrome. Ann Clin Biochem. 2003; 40(Pt3):219-231. doi: 10.1258/000456303321610501.

33. Berktold L, von Kaisenberg CS, Hillemanns P, Vaske B, Schmidt P. Analysis of the impact of PAPP-A, free $\beta-h C G$ and nuchal translucency thickness on the advanced first trimester screening. Arch Gynecol Obstet. 2013; 287(3):413-420. doi: 10.1007/s00404012-2585-y.

34. Scala C, Morlando M, Familiari A, Leone Roberti Maggiore U, Ferrero S, D'Antonio F, et al. Fetal Tricuspid Regurgitation in the First Trimester as a Screening Marker for Congenital Heart Defects: Systematic Review and Meta-Analysis. Fetal Diagn Ther. 2017; 42(1):1-8. doi: 10.1159/000455947.

35. Padula F, Cignini P, Giannarelli D, Brizzi C, Coco C, D'Emidio L, et al. Retrospective study evaluating the performance of a first-trimester combined screening for trisomy 21 in an Italian unselected population. J Prenat Med [Internet]. 2014 [consultado diciembre 2020]; 8(3-4):50-56. Disponible en: https:/www.ncbi.nlm. nih.gov/pmc/articles/PMC4510564/pdf/50-56.pdf
36. Falcon O, Auer M, Gerovassili A, Spencer K, Nicolaides KH. Screening for trisomy 21 by fetal tricuspid regurgitation, nuchal translucency and maternal serum free beta-hCG and PAPP-A at $11+0$ to $13+6$ weeks. Ultrasound Obstet Gynecol. 2006; 27(2):151-155. doi: 10.1002/uog.2699.

37. Maiz N, Nicolaides KH. Ductus venosus in the first trimester: contribution to screening of chromosomal, cardiac defects and monochorionic twin complications. Fetal Diagn Ther. 2010; 28(2):65-71. doi: 10.1159/000314036.

38. Tang Y, Luo H, Mu D, Yang T, Zhu Q, Yang F, et al. Early diagnosis of trisomy 21, trisomy 18 and trisomy 13 using nuchal translucency thickness and ductus venosus blood flow waveform in West China. Mol Med Rep. 2019; 19(2):1349-1355. doi: 10.3892/ mmr.2018.9756.

39. Florjański J, Fuchs T, Zimmer M, Homola W, Pomorski M, Blok D. The role of ductus venosus Doppler flow in the diagnosis of chromosomal abnormalities during the first trimester of pregnancy. Adv Clin Exp Med [Internet]. 2013 [consultado noviembre de 2020]; 22(3):395-401. Disponible en: https://advances.umw. edu.pl/pdf/2013/22/3/395.pdf

40. Sosa C, Gómez L, Bermúdez C, Pérez-Wulff J. Aumento de la translucencia nucal y flujo reverso del ductus venoso en cardiopatía congénita. Reporte de un caso en Caracas, Venezuela. Rev Colomb Obstet Ginecol. 2008; 59(1): 57-61. https://doi.org/10.18597/ $\operatorname{rcog} .432$

41. Ghaffari SR, Tahmasebpour AR, Jamal A, Hantoushzadeh S, Eslamian L, Marsoosi V, et al. Firsttrimester screening for chromosomal abnormalities by integrated application of nuchal translucency, nasal bone, tricuspid regurgitation and ductus venosus flow combined with maternal serum free $\beta-\mathrm{hCG}$ and PAPP-A: a 5-year prospective study. Ultrasound Obstet Gynecol. 2012; 39(5):528-534. doi: 10.1002/uog.10051.

42. Antsaklis P, Fasoulakis Z, Theodora M, Diakosavvas M, Kontomanolis EN. Association of Low Maternal Pregnancy-associated Plasma Protein A with Adverse Perinatal Outcome. Cureus. 2019; 11(6):e4912. doi: $10.7759 /$ cureus.4912.

43. Kagan KO, Cicero S, Staboulidou I, Wright D, Nicolaides KH. Fetal nasal bone in screening for trisomies 21, 18 and 13 and Turner syndrome at 11-13 weeks of gestation. Ultrasound Obstet Gynecol. 2009; 33(3):259-264. doi: 10.1002/uog.6318. 
44. Abele H, Wagner P, Sonek J, Hoopmann M, Brucker $\mathrm{S}$, Artunc-Ulkumen $\mathrm{B}$, et al. First trimester ultrasound screening for Down syndrome based on maternal age, fetal nuchal translucency and different combinations of the additional markers nasal bone, tricuspid and ductus venosus flow. Prenat Diagn. 2015; 35(12):1182-1186. doi: 10.1002/pd.4664.

45. Poureisa M, Daghighi MH, Mazaheri Khameneh R, Salehi Majd S. Fetal Nasal Bone Status In Iranian Women Undergoing First-Trimester Screening For Trisomy 21: A Review and an Observational Study. Iran J Radiol. 2015; 12(4):e11905. doi: 10.5812/ iranjradiol.11905.

46. Bilardo CM, Timmerman E, Pajkrt E, van Maarle M. Increased nuchal translucency in euploid fetuses - what should we be telling the parents? Prenat Diagn. 2010; 30(2):93-102. doi: 10.1002/pd.2396.

47. Bijok J, Ziora-Jakutowicz K, Ilnicka A, Pawłowska $\mathrm{B}$, Jóźwiak A, et al. [Increased nuchal translucency in chromosomally normal fetuses and pregnancy outcomes--a retrospective study]. Ginekol Pol. 2013; 84(3):172-179. Polish. doi: 10.17772/gp/1559..

48. Maymon R, Weinraub Z, Herman A. Pregnancy outcome of euploid fetuses with increased nuchal translucency: how bad is the news? J Perinat Med. 2005; 33(3):191-198. doi: 10.1515/JPM.2005.036.

49. Socolov D, Socolov R, Gorduza VE, Butureanu T, Stanculescu R, Carauleanu A, et al. Increased nuchal translucency in fetuses with a normal karyotypediagnosis and management: An observational study. Medicine (Baltimore). 2017; 96(29):e7521. doi: 10.1097/MD.0000000000007521.

50. Tahmasebpour A, Rafiee NB, Ghaffari S, Jamal A. Increased nuchal translucency and pregnancy outcome. Iran J Public Health [Internet]. 2012 [consultado noviembre de 2020]; 41(11): 92-97. Disponible en: https://ijph.tums.ac.ir/index.php/ijph/article/view/2492

51. Smrcek JM, Gembruch U, Krokowski M, Berg C, Krapp M, Geipel A, et al. The evaluation of cardiac biometry in major cardiac defects detected in early pregnancy. Arch Gynecol Obstet. 2003; 268(2):94-101. doi: 10.1007/s00404-002-0358-8.

52. Moczulska H, Janiak K, Słodki M, Respondek-Liberska M. Ultrasound and echocardiographic findings obtained in the second and third trimesters of gestation in fetuses with normal karyotype and increased nuchal translucency. J Ultrason. 2013; 13(52):21-30. doi: 10.15557/JoU.2013.0002.
53. Moczulska H, Janiak K, Słodki M, Respondek-Liberska M. Ultrasound and echocardiographic findings obtained in the second and third trimesters of gestation in fetuses with normal karyotype and increased nuchal translucency. J Ultrason. 2013; 13(52):21-30. doi: 10.15557/JoU.2013.0002.

54. Egbe A, Uppu S, Stroustrup A, Lee S, Ho D, Srivastava S. Incidences and sociodemographics of specific congenital heart diseases in the United States of America: an evaluation of hospital discharge diagnoses. Pediatr Cardiol. 2014; 35(6):975-982. doi: 10.1007/ s00246-014-0884-8.

55. Şahin Uysal N, Gülümser Ç, Yılmaz Çelik Z, Yanık FB. Increased nuchal translucency and pregnancy outcomes: experience of Başkent University Ankara Hospital. Turk J Obstet Gynecol. 2019; 16(2):100-106. doi: 10.4274/tjod.galenos.2019.51482.

56. Bromley B, Shipp TD, Lyons J, Navathe RS, Groszmann Y, Benacerraf BR. Detection of fetal structural anomalies in a basic first-trimester screening program for aneuploidy. J Ultrasound Med. 2014; 33(10):17371745. doi: 10.7863/ultra.33.10.1737.

57. Pinto NM, Nelson R, Puchalski M, Metz TD, Smith KJ. Cost-effectiveness of prenatal screening strategies for congenital heart disease. Ultrasound Obstet Gynecol. 2014; 44(1):50-57. doi: 10.1002/uog.13287.

58. Li L, Fu F, Li R, Liu Z, Liao C. Prenatal diagnosis and pregnancy outcome analysis of thickened nuchal fold in the second trimester. Medicine (Baltimore). 2018; 97(46):e13334. doi: 10.1097/MD.0000000000013334.

59. Hunter LE, Simpson JM. Prenatal screening for structural congenital heart disease. Nat Rev Cardiol. 2014; 11(6):323-334. doi: 10.1038/nrcardio.2014.34.

60. Baś-Budecka E, Perenc M, Sieroszewski P. [The role of fetal nuchal translucency (NT) and ductus venosus blood flow (DV) in the detection of congenital heart defects]. Ginekol Pol [Internet]. 2010 [consultado noviembre 2020]; 81(4):272-276. Polish. Disponible en: https://journals.viamedica.pl/ginekologia_polska/ article/view/46484/33271

61. de Mooij YM, Haak MC, Bartelings MM, Twisk JW, Gittenberger-de Groot A, van Vugt JM, et al. Abnormal ductus venosus flow in first-trimester fetuses with increased nuchal translucency: relationship with the type of cardiac defect? J Ultrasound Med. 2010; 29(7):1051-8. doi: 10.7863/jum.2010.29.7.1051. 


\section{IMPORTANCIA CLÍNICA DE LA EVALUACIÓN ECOGRÁFICA \\ DE LA TRANSLUCENCIA NUCAL FETAL}

62. Ozer O, Sayın CN, Varol FG. The assessment of nuchal translucency and serum markers for down syndrome screening with ductus venosus Doppler measurements in the first trimester. J Turk Ger Gynecol Assoc. 2010; 11(4):194-198. doi: 10.5152/jtgga.2010.36.

63. Zhang Z, Hu T, Wang J, Li Q, Wang H, Liu S. Prenatal Diagnostic Value of Chromosomal Microarray in Fetuses with Nuchal Translucency Greater than $2.5 \mathrm{~mm}$. Biomed Res Int. 2019; 2019:6504159. doi: 10.1155/2019/6504159.

64. Miltoft CB, Ekelund CK, Hansen BM, Lando A, Petersen $\mathrm{OB}$, Skovbo $\mathrm{P}$, et al. Increased nuchal translucency, normal karyotype and infant development. Ultrasound Obstet Gynecol. 2012; 39(1):28-33. doi: 10.1002/ uog. 10060.

65. Emami-Moghaddam A, Barati M, Amirpour R, Shojaei K. Prenatal and postnatal echocardiography in NT fetuses with normal karyotype. J Family Med Prim Care. 2019; 8(8):2667-2670. doi: 10.4103/jfmpc. jfmpc_395_19.

66. Bardi F, Bosschieter P, Verheij J, Go A, Haak M, Bekker $\mathrm{M}$, et al. Is there still a role for nuchal translucency measurement in the changing paradigm of first trimester screening? Prenat Diagn. 2020; 40(2):197-205. doi: 10.1002/pd.5590.

67. Monni G, Iuculano A, Zoppi MA. Screening and Invasive Testing in Twins. J Clin Med. 2014; 3(3):865882. doi: 10.3390/jcm3030865.

68. Fosler L, Winters P, Jones KW, Curnow KJ, Sehnert AJ, Bhatt S, et al. Aneuploidy screening by noninvasive prenatal testing in twin pregnancy. Ultrasound Obstet Gynecol. 2017; 49(4):470-477. doi: 10.1002/ uog. 15964.

69. Spencer K. Screening for trisomy 21 in twin pregnancies in the first trimester using free beta-hCG and PAPP-A, combined with fetal nuchal translucency thickness. Prenat Diagn. 2000; 20(2):91-95. doi: $10.1002 /$ (sici)1097-0223(200002)20:2<91::aidpd759>3.0.co;2-x.

70. Cleary-Goldman J, Berkowitz RL. First trimester screening for Down syndrome in multiple pregnancy. Semin Perinatol. 2005; 29(6):395-400. doi: 10.1053/j. semperi.2006.01.002.
71. Platt LD. First-trimester risk assessment: twin gestations. Semin Perinatol. 2005; 29(4):258-262. doi: 10.1053/j.semperi.2005.06.003.

72. Maruotti GM, Saccone G, Morlando M, Martinelli P. First-trimester ultrasound determination of chorionicity in twin gestations using the lambda sign: a systematic review and meta-analysis. Eur J Obstet Gynecol Reprod Biol. 2016; 202:66-70. doi: 10.1016/j. ejogrb.2016.04.023.

73. Goncé A, Borrell A, Meler E, Arigita M, Martínez JM, Botet F, et al. Prevalence and perinatal outcome of dichorionic and monochorionic twins with nuchal translucency above the 99(th) percentile and normal karyotype. Ultrasound Obstet Gynecol. 2010; 35(1):1418. doi: 10.1002/uog.7498.

74. Prats P, Rodríguez I, Comas C, Puerto B. First trimester risk assessment for trisomy 21 in twin pregnancies combining nuchal translucency and first trimester biochemical markers. Prenat Diagn. 2012; 32(10):927932. doi: 10.1002/pd.3934.

75. Stagnati V, Zanardini C, Fichera A, Pagani G, Quintero RA, Bellocco R, et al. Early prediction of twin-to-twin transfusion syndrome: systematic review and metaanalysis. Ultrasound Obstet Gynecol. 2017; 49(5):573582. doi: 10.1002/uog.15989.

76. Maslovitz S, Yaron Y, Fait G, Gull I, Wolman I, Jaffa A, et al. Feasibility of nuchal translucency in triplet pregnancies. J Ultrasound Med. 2004; 23(4):501-504. doi: 10.7863/jum.2004.23.4.501.

77. Maymon R, Dreazen E, Tovbin Y, Bukovsky I, Weinraub Z, Herman A. The feasibility of nuchal translucency measurement in higher order multiple gestations achieved by assisted reproduction. Hum Reprod. 1999; 14(8):2102-2105. doi: 10.1093/humrep/14.8.2102.
Recibido 11 de enero de 2021 Aprobado 3 de abril de 2021 